Research paper

\title{
Identification and characterization of plasmid-mediated quinolone resistance determinants in Enterobacteriaceae isolated from healthy poultry in Brazil
}

\author{
Joseane Cristina Ferreira $^{\mathrm{a}}$, Rafael Antonio Casarin Penha Filho ${ }^{\mathrm{b}}$, Ana Paula Yorika Kuaye ${ }^{\mathrm{a}}$, \\ Leonardo Neves Andrade ${ }^{\mathrm{a}}$, Angelo Berchieri Junior ${ }^{\mathrm{b}}$, Ana Lúcia da Costa Darini ${ }^{\mathrm{a}}$, \\ a School of Pharmaceutical Sciences of Ribeirão Preto, São Paulo University (USP), Ribeirão Preto, SP 14040-903, Brazil \\ ${ }^{\mathrm{b}}$ School of Agricultural and Veterinary Sciences, São Paulo State University (UNESP), Jaboticabal, SP 14884-900, Brazil
}

\section{A R T I C L E I N F O}

\section{Keywords:}

qnr

PMQR

Food-producing animal

Chicken

ColE-like plasmid

\begin{abstract}
A B S T R A C T
The expression of plasmid-mediated quinolone resistance (PMQR) genes confers low-level quinolone and fluoroquinolones resistance alone. However, the association to chromosomal resistance mechanisms determines an expressively higher resistance in Enterobacteriaceae. These mechanisms are horizontally disseminated within plasmids and have contributed to the emergence of bacteria with reduced susceptibility or resistant to therapies worldwide. The epidemiological characterization of PMQR dissemination is highly relevant in the scientific and medical context, to investigate the dissemination within enterobacteria, from different populations, including humans and food-producing animals. In the present study, 200 Enterobacteriaceae isolates were harvested from poultry with cloacal swabs and identified as Escherichia coli (90.5\%), Escherichia fergusonii (5.5\%), Klebsiella oxytoca (2.5\%) and Klebsiella pneumoniae (1.5\%). Among isolates evaluated, 46 (23\%) harboured PMQR genes including $q n r \mathrm{~B}(43 / 200)$, qnrS (2/200) and $a a c\left(6^{\prime}\right)-I b$-cr (1/200). All isolates carrying PMQR genes showed multidrug-resistance phenotype. The $36 \mathrm{E}$. coli isolates showed 18 different PFGE types. All $E$. fergusonii isolates showed the same PFGE type. The two Klebsiella oxytoca belonged to two different PFGE types. The phylogenetic groups A, B1, and D were found among the E. coli harboring PMQR genes. Based on the phylogenetic analysis and PFGE, the population structure of $E$. coli isolates was diverse, even within the same farm. All isolates carrying $q n r B$ and $q n r S$ genes also harboured ColE-like plasmids. The Southern blot hybridization using the S1-PFGE revealed that the $q n r \mathrm{~B}$ genes were located on low molecular weight plasmids, smaller than $10 \mathrm{~Kb}$. Resistance plasmids were sequenced and showed 100\% identity with plasmid pPAB19-3. The association of PMQR genes with mobile genetic elements, such as transferable plasmids, favours the selection and dissemination of (fluoro) quinolones resistant bacteria among food-producing animals, and may play an important role in the current increased prevalence of resistant bacteria in different environments reported worldwide.
\end{abstract}

\section{Introduction}

The introduction of fluoroquinolones in clinical therapies occurred in the 1980s and the advantage of oral administration associated with the bactericidal activity against Gram-negative microorganisms contributed to the frequent election of this class of antimicrobials to treat infections caused by Escherichia coli, Salmonella and other Enterobacteriaceae in humans and animals, to date (Dalhoff, 2012; Vanni et al., 2014; Zawack et al., 2016).

Among different fluoroquinolone resistance mechanisms, chromosomal mutations in gyrA and/or parC genes in quinolone-resistance- determining region (QRDR), may change the antibiotic target site, consequently reducing the therapeutic efficacy. This mechanism associated to the expression of plasmid-mediated quinolone resistance (PMQR) genes increases the resistance level of the bacteria, frequently reaching impracticable minimal inhibitory concentration (MIC) (Harada and Asai, 2010; Gagliotti et al., 2008). The main PMQR determinants reported are the $q n r$ genes ( $q n r \mathrm{~A}, q n r \mathrm{~B}, q n r \mathrm{C}, q n r \mathrm{D}$ and $q n r \mathrm{~S}$ ) that mediate resistance to quinolones by protecting the antimicrobial target protein, type II DNA topoisomerases, from the action of these antimicrobials (Rodriguez-Martinez et al., 2011). Moreover, there are other PMQR determinants, for example, $a a c\left(6^{\prime}\right)-I b-c r$ genes, which

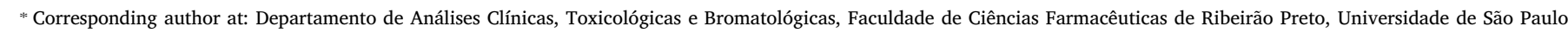
(USP), Ribeirão Preto, SP 14040-903, Brazil.

E-mail address: aldarini@fcfrp.usp.br (A.L.d.C. Darini). 
codify acetyltransferase, an aminoglycoside modifying enzyme able to acetylate fluoroquinolones due to the "-cr variant" and the QepA and OqxAB efflux pump proteins, that decrease susceptibility to hydrophilic fluoroquinolones. The expression of these genes associated with other resistance mechanisms, such as alteration of the target site, porin alterations, and overexpression of chromosomal efflux systems have been contributing to the increased level of quinolone resistance as well as the selection of resistant clones in enterobacteria (Strahilevitz et al., 2009; Tamang et al., 2011).

The prevalence of $q n r \mathrm{~B}$ genes is higher than other $q n r$ genes in Enterobacteriaceae isolates from human, animal, and environment (Poirel et al., 2012) with about 80 different alleles assigned in the Lahey website (http://www.lahey.org/qnrStudies/), currently. The qnrB19 gene has been reported worldwide in Enterobacteriaceae isolated from healthy humans, clinical infections and food-producing animals (Dionisi et al., 2009; Karczmarczyk et al., 2010; Pallecchi et al., 2011). This gene has been reported in plasmids IncN, IncX and small rollingcircle-replication (RCR) plasmids (Carattoli, 2013). The Xer-mediated site-specific recombination is often identified in RCR plasmids, especially ColE1, and has been suggested as the source of acquisition of the resistance gene (Tran et al., 2012). Most of the qnr-carrying plasmids share high similarity with ColE1 replicon (Carattoli, 2013). This study was conducted to evaluate the prevalence of PMQR determinants and characterize plasmids harboring these genes in Enterobacteriaceae isolated from poultry in Brazil.

\section{Materials and methods}

\subsection{Isolation of bacteria}

From 2011 to 2012, a total of 200 isolates were screened from 200 healthy chickens from two different poultry Farms in Sao Paulo State, Brazil. The procedure for sampling was previously approved by the Institutional Animal Use and Ethics Committee (Proc. n. 12.1.248.53.7). The cloacal swab samples were seeded onto MacConkey agar plates containing ciprofloxacin $(2 \mu \mathrm{g} / \mathrm{mL})$ and incubated for $24 \mathrm{~h} /$ $37^{\circ} \mathrm{C}$. Colonies were identified by biochemical standard methods, confirmed by API 20E test (BioMérieux, France) and, in some cases, the identification was performed by VITEK $^{\oplus} 2$ (BioMérieux, France).

\subsection{Screening of $P M Q R$ genes by multiplex PCR}

The total genomic DNA of the isolates was extracted as described previously (Bolano et al., 2001), and used in the PCR for the identification of the PMQR genes in all isolates. The genes $q n r \mathrm{~A}, q n r \mathrm{~B}, q n r \mathrm{~S}$ (Cattoir et al., 2007), qnrC, qnrD (Wang et al., 2009), acc(6')-Ib-cr, qepA and oqxAB (Minarini et al., 2008) were searched as previously described. Both strands of the amplicons were sequenced using the ABI 3730 sequencer (Applied Biosystems, USA) with the same primers used for PCR.

\subsection{Antimicrobial susceptibility}

The antimicrobial susceptibility of $E$. coli isolates with PMQR genes were assessed by agar disk diffusion method following the recommendations by Clinical Laboratory Standards Institute (CLSI, 2002), using breakpoints recommended by CLSI (2013). Fifteen antimicrobial drugs (Oxoid, UK) were tested: amoxillin/clavulanic acid $(20 / 10 \mu \mathrm{g})$, piperacillin/tazobactam $(100 / 10 \mu \mathrm{g})$, cefotaxime $(30 \mu \mathrm{g})$, ceftazidime $(30 \mu \mathrm{g})$, cefepime $(30 \mu \mathrm{g})$, cefoxitin $(30 \mu \mathrm{g})$, aztreonam $(30 \mu \mathrm{g})$, ertapenem $(10 \mu \mathrm{g})$, nalidixic acid $(30 \mu \mathrm{g})$, ciprofloxacin $(5 \mu \mathrm{g})$, levofloxacin $(5 \mu \mathrm{g})$, enrofloxacin $(5 \mu \mathrm{g})$, tetracycline $(30 \mu \mathrm{g})$, gentamicin $(10 \mu \mathrm{g})$, trimethoprim-sulfamethoxazole $(1.25 / 23.75 \mu \mathrm{g})$ and chloramphenicol $(30 \mu \mathrm{g})$. Multidrug-resistance (MDR) phenotype was characterized by non-susceptibility or resistance to at least one agent in three or more antimicrobial classes (Canton and Ruiz-Garbajosa, 2011;
Magiorakos et al., 2012).

\subsection{Pulsed-field gel electrophoresis (PFGE)}

Genomic DNA of $q n r$ positive $E$. coli isolates were digested with restriction enzyme $\mathrm{XbaI}$ (Fermentas, USA) and the macrorestriction separation was carried out using pulsed-field gel electrophoresis (PFGE) in the CHEF-DRIII System (Bio-Rad Laboratories, USA). The assay was performed in 1,2\% agarose PFGE gels at $6.0 \mathrm{~V} / \mathrm{cm}$ with an initial/final switch time of $10 / 40 \mathrm{~s}$ and an angle of $120^{\circ}$ at $14{ }^{\circ} \mathrm{C}$ for $24 \mathrm{~h}$. Macrorestriction patterns were analysed using the BioNumerics 5.0 software (Applied Maths, USA). Relationship among PFGE-types was analysed by the Dice similarity index to obtain a dendrogram with clusters. The dendrogram was constructed using the unweighted-pair group method using average linkage algorithm (UPGMA). The minimum threshold value for similar PFGE patterns was defined at $85 \%$.

\subsection{Phylogenetic analysis}

The phylogenetic groups were determined according to the previously described method (Clermont et al., 2000), classifying isolates to one of the four phylogenetic groups (A, B1, B2, or D) based on the presence of chuA, yjaA genes and TspE4C2 DNA fragment.

\subsection{Plasmid replicon typing}

Plasmids were investigated by PCR-based Replicon Typing (PBRT) method using primers for 18 major incompatibility (Inc) groups described (FIA, FIB, FIC, HI2, I1-I, L/M, N, P, W, T, A/C, K, B/O, X, Y, F, FIIA, HI1) among Enterobacteriaceae as described previously (Carattoli et al., 2005). ColE plasmids were also searched by previously described method (Garcia-Fernandez et al., 2009). The Inc groups were determined in parental strains to characterize all plasmids, including the non-conjugative.

\subsection{Determination of the $P M Q R$}

To determine the plasmids harboring the PMQR genes, PFGE was performed, after digestion with $S 1$ nuclease (S1-PFGE). The Lambda Ladder PFG Marker and Low Range PFG Marker (Biolabs, USA) were used as size standards. The Southern blot and hybridization were performed using DNA probes specific for quinolone resistance genes prepared with AlkPhos Direct Labeling and Detection System with CDPStar (GE Healthcare Life Sciences, USA) (Sambrook et al., 1989).

\subsection{Characterization of ColE-like plasmids}

The plasmids were extracted using PureYield ${ }^{\mathrm{TM}}$ Plasmid Miniprep System (Promega, USA), according to manufacturer instructions. The ColE-like plasmids were characterized using the PCR-based method described elsewhere (Pallecchi et al., 2010). The complete sequence of these plasmids was obtained by Sanger sequencing method (ABI 3730 sequencer, Applied Biosystems, USA) and analysed using Chromas Pro (Technelysium, Australia) and BLAST (http://www.ncbi.nlm.nih.gov/ BLAST/) softwares.

\section{Results and discussion}

Antimicrobial resistance has been exponentially noticed in Enterobacteriaceae isolated from infected (Dalhoff, 2012) and colonized humans (Woerther et al., 2013) and also from food and food-producing animals (Bardon et al., 2013; Clemente et al., 2015; Liebana et al., 2013; Szmolka et al., 2011; Tamang et al., 2011). Two hundred ciprofloxacin resistant Enterobacteriaceae isolates were isolated from 200 cloacal swabs streaked on MacConkey agar plates, selecting one colony 
Table 1

Phenotypic and genotypic characteristics of PMQR-harboring E. coli isolates from poultry.

\begin{tabular}{|c|c|c|c|c|c|c|c|}
\hline Isolates & Species & Farm & Resistance & PMQR gene & Plasmid family & Phylogenetic group & PFGE-type \\
\hline $157 a$ & E. coli & 2 & AMC, CTX, CAZ, FOX, NAL, CIP, LEV, GEN, TET, SXT & qnrB19 & ColE, HI1, I1, F & A & A \\
\hline 167 & E. coli & 2 & AMC, CTX, FOX, NAL, CIP, LEV, GEN, TET, SXT & qnrB19 & ColE, FIB, F & A & A \\
\hline $166 \mathrm{a}$ & E. coli & 2 & AMC, CTX, CAZ, FOX, NAL, CIP, LEV, GEN, TET, SXT & qnrB19 & ColE, FIB, F & A & B \\
\hline 109 & E. coli & 2 & AMC, CTX, CAZ, FOX, NAL, CIP, LEV, TET, SXT & qnrB19 & $\overline{\mathrm{ColE}}, \mathrm{K}$ & A & $\mathrm{C}$ \\
\hline 107 & E. coli & 2 & AMC, CTX, CAZ, FOX, NAL, CIP, LEV, TET, SXT, CHL & qnrB19 & $\overline{\mathrm{ColE}}, \mathrm{FIB}, \mathrm{F}$ & $\mathrm{D}$ & $\mathrm{D}$ \\
\hline 110 & E. coli & 2 & AMC, CTX, CAZ, FOX, NAL, CIP, LEV, TET, SXT, CHL & qnrB19 & ColE, F & $\mathrm{D}$ & $\mathrm{E}$ \\
\hline 112 & E. coli & 2 & AMC, CTX, CAZ, FOX, NAL, CIP, LEV, TET, CHL & qnrB19 & ColE, FIB, F & $\mathrm{D}$ & $\mathrm{E}$ \\
\hline 115 & E. coli & 2 & AMC, CTX, CAZ, ATM, FOX, NAL, CIP, LEV, TET, CHL & qnrB19 & ColE, FIB, F & $\mathrm{D}$ & $\mathrm{E}$ \\
\hline $125 \mathrm{a}$ & E. coli & 2 & AMC, CTX, NAL, CIP, LEV, TET, CHL & qnrB19 & ColE, FIB, F & $\mathrm{D}$ & $\mathrm{E}$ \\
\hline 127 & E. coli & 2 & AMC, CTX, CAZ, ATM, FOX, NAL, CIP, LEV, TET, CHL & qnrB19 & ColE, FIB, F & $\mathrm{D}$ & $\mathrm{E}$ \\
\hline 135 & E. coli & 2 & AMC, CTX, CAZ, ATM, FOX, NAL, CIP, LEV, TET, CHL & qnrB19 & $\overline{\mathrm{ColE}}, \mathrm{FIB}, \mathrm{F}$ & $\mathrm{D}$ & $\mathrm{E}$ \\
\hline 138 & E. coli & 2 & AMC, CTX, CAZ, ATM, FOX, NAL, CIP, LEV, TET, CHL & qnrB19 & ColE, FIB, F & $\mathrm{D}$ & $\mathrm{E}$ \\
\hline $141 b$ & E. coli & 2 & AMC, CTX, FOX, NAL, CIP, LEV, TET, CHL & qnrB19 & ColE, FIB, F & $\mathrm{D}$ & $\mathrm{E}$ \\
\hline 148 & E. coli & 2 & AMC, CTX, CAZ, ATM, FOX, NAL, CIP, LEV, TET, CHL & qnrB19 & ColE, FIB, F & $\mathrm{D}$ & $\mathrm{E}$ \\
\hline 149 & E. coli & 2 & AMC, CTX, CAZ, ATM, FOX, NAL, CIP, LEV, TET, CHL & qnrB19 & $\overline{\mathrm{ColE}}, \mathrm{FIB}, \mathrm{F}$ & $\mathrm{D}$ & $\mathrm{E}$ \\
\hline $114 \mathrm{~b}$ & E. coli & 2 & AMC, CTX, CAZ, ATM, FOX, NAL, CIP, LEV, TET, CHL & qnrB19 & ColE, FIB, F & $\mathrm{D}$ & $\mathrm{E}$ \\
\hline 122 & E. coli & 2 & AMC, CTX, CAZ, ATM, FOX, NAL, CIP, LEV, TET, CHL & qnrB19 & ColE FIB, F & $\mathrm{D}$ & $\mathrm{E}$ \\
\hline 176 & E. coli & 2 & AMC, CTX, CAZ, FOX, NAL, CIP, LEV, GEN, TET, SXT & qnrB19 & ColE, FIA, FIB, F & $\mathrm{D}$ & $\mathrm{F}$ \\
\hline $39 a$ & E. coli & 1 & AMC, CTX, CAZ, FOX, NAL, CIP, LEV & qnrB19 & $\overline{\mathrm{ColE}}, \mathrm{K}, \mathrm{B} / \mathrm{O}$ & A & G \\
\hline 74 & E. coli & 1 & AMC, CTX, CAZ, ATM, FOX, NAL, CIP, LEV, TET & qnrB19 & ColE, $\mathrm{K}$ & A & G \\
\hline 91 & E. coli & 1 & AMC, CTX, CAZ, FOX, NAL, CIP, LEV & qnrB5 & $\overline{\mathrm{ColE}}, \mathrm{K}$ & A & G \\
\hline $123 b$ & E. coli & 2 & AMC, CTX, NAL, CIP, LEV, GEN, TET & qnrB19 & $\overrightarrow{\mathrm{ColE}}, \mathrm{K}$ & A & $\mathrm{H}$ \\
\hline 136 & E. coli & 2 & AMC, CTX, FOX, NAL, CIP, LEV, GEN, TET & qnrB19 & $\overline{\text { ColE, }} \mathrm{K}$ & A & $\mathrm{H}$ \\
\hline 146 & E. coli & 2 & AMC, CTX, NAL, CIP, LEV, GEN, TET & qnrB19 & $\overline{\mathrm{ColE}}, \mathrm{K}$ & A & $\mathrm{H}$ \\
\hline 105 & E. coli & 2 & AMC, CTX, FOX, NAL, CIP, LEV, GEN, TET & qnrB19 & $\overline{\mathrm{ColE}}$, FIA & A & $\mathrm{H}$ \\
\hline 147 & E. coli & 2 & AMC, CTX, FOX, NAL, CIP, LEV, GEN, TET & qnrB19 & ColE, K & A & $\mathrm{H}$ \\
\hline 86 & E. coli & 1 & AMC, CTX, ATM, NAL, CIP, LEV, GEN, TET & qnrB5 & ColE, FIB, FIC, F & A & $\mathrm{N}$ \\
\hline 106 & E. coli & 2 & AMC, CTX, CAZ, FOX, NAL, CIP, LEV, GEN, TET, SXT & qnrB19 & $\overline{\text { ColE, }}$ FIA, F & $\mathrm{D}$ & I \\
\hline 143 & E. coli & 2 & AMC, CTX, FOX, NAL, CIP, LEV, SXT & qnrB19 & ColE, $\mathrm{K}$ & A & $\mathrm{J}$ \\
\hline 12 & E. coli & 1 & AMC, CTX, CAZ, ATM, FOX, NAL, CIP, LEV, CHL & qnrB19 & $\overline{\text { ColE}}$, FIC, K, F & A & $\mathrm{K}$ \\
\hline 37 & E. coli & 1 & AMC, CTX, CAZ, ATM, FOX, NAL, CIP, LEV, TET & qnrB19 & $\overline{\mathrm{ColE}}, \mathrm{K}, \mathrm{B} / \mathrm{O}$ & A & $\mathrm{L}$ \\
\hline 89 & E. coli & 1 & AMC, CTX, CAZ, ATM, FEP, NAL, CIP, LEV, TET, CHL & qnrB5 & ColE, $\mathrm{F}$ & B1 & M \\
\hline 82 & E. coli & 1 & AMC, CTX, FEP, NAL, CIP, TET & qnrB5 & ColE, I1, FIB, F & A & $\mathrm{O}$ \\
\hline 49a & E. coli & 1 & AMC, CTX, CAZ, ATM, FEP, NAL, CIP, LEV, TET, SXT, CHL & $\operatorname{aac}\left(6^{\prime}\right)-\mathrm{Ib}-\mathrm{cr}$ & FIB, F,Y & B1 & $\mathrm{P}$ \\
\hline 01 & E. coli & 1 & AMC, CTX, CAZ, ATM, FEP, NAL, CIP, TET & qnrS1 & ColE, I1, FIB, F, A/C & B1 & $\mathrm{Q}$ \\
\hline 93 & E. coli & 1 & AMC, CTX, ATM, FEP, NAL, CIP, LEV, TET, SXT, CHL & qnrS1 & ColE, HI1, I1, FIB, F & A & $\mathrm{R}$ \\
\hline
\end{tabular}

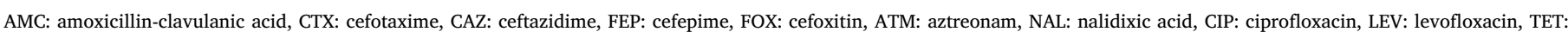
tetracycline, CHL: chloramphenicol, SXT: trimethoprim-sulfamethoxazole, GEN: gentamicin. The plasmids that carried PMQR genes are underlined.

per plate after incubation. Among the identified isolates, $90.5 \%$ were $E$. coli (181/200), 5.5\% Escherichia fergusonii (11/200), 2.5\% Klebsiella oxytoca $(5 / 200)$ and $1.5 \%$ Klebsiella pneumoniae $(3 / 200)$.

Out of 200 Enterobacteriaceae isolates examined in this study, $23 \%$ (46/200) carried PMQR genes including qnrB (43/200), qnrS (2/200) and $a a c\left(6^{\prime}\right)-I b-c r(1 / 200)$. Among Qnr-producing isolates carrying qnrB, 33 were $E$. coli isolates ( $q n r \mathrm{~B} 19, \mathrm{n}=29 ;$ qnrB5, $\mathrm{n}=4$ ), 7 E. fergusonii (qnrB19), 2 K. oxytoca (qnrB19) and 1 K. pneumoniae (qnrB19). The $q n r S 1$ was found in two $E$. coli isolates and $a a c\left(6^{\prime}\right)-I b$-cr gene was found in one $E$. coli isolate (Table 1). None isolate was positive for $q n r \mathrm{~A}, q n r \mathrm{C}$, $q n r \mathrm{D}$, qepA and oqxAB genes. In our work, the most prevalent PMQR determinant was $q n r B(n=43)$, found in different bacterial species. Other research groups have also reported this gene as the most frequent PMQR determinant, followed by qnrS (Cattoir and Nordmann, 2009; Rodriguez-Martinez et al., 2011; Strahilevitz et al., 2009). The qnrB19 ( $\mathrm{n}=39$ ) was the most prevalent gene detected in our study, followed by $q n r B 5(\mathrm{n}=4)$. The gene $q n r B 19$ was reported in other countries, in E. coli from chickens, however, in most cases, in lower prevalence (Ben Sallem et al., 2014; Fortini et al., 2011; Literak et al., 2013; Oh et al., 2016) than described in the present study. Our results show a high prevalence of $q n r \mathrm{~B} 19$ in poultry produced for meat consumption. There is few data about PMQR determinants either in humans or in animals in Brazil, therefore the prevalence of these genes is poorly understood. Another study in Brazil, also reported the gene qnrB19 carried by ColElike plasmids in four ExPEC isolates from poultry (Cunha et al., 2017).

In this study, all isolates carrying PMQR genes (46/46) showed MDR phenotype. Among these isolates, the resistance to antibiotics tested was: amoxicillin-clavulanic acid (100\%), cefotaxime (100\%), ceftazidime (56\%), cefepime (15\%), cefoxitin (63\%), aztreonam (41\%), nalidixic acid (NAL 100\%), ciprofloxacin (CIP 91\%), levofloxacin (LEV $82 \%$ ), enrofloxacin (ENR 100\%), tetracycline (89\%), gentamicin (24\%), trimethoprim-sulfamethoxazole (63\%), and chloramphenicol (46\%). None isolate showed resistance to piperacillin/tazobactam and ertapenem (Tables 1 and 2). NAL, CIP and LEV are mainly used to treat urinary tract infections and respiratory infections in human patients. However, ENR is a fluoroquinolone exclusively developed for use in veterinary medicine (Lopez-Cadenas et al., 2013) and has been extensively used as a prophylactic measure in poultry farms, to reduce intestinal infections by Salmonella and other pathogens, which may have contributed for the selection of fluoroquinolone resistance among these animals. Furthermore, the oral use of ENR also contributes for selection of resistance to non-quinolone antimicrobials in commensal $E$. coli isolated from chickens (Jurado et al., 2015). The use of other antibiotic classes, such as beta-lactam antibiotics, could advantage coselection of fluoroquinolones non-susceptible $E$. coli from retail poultry (Ingram et al., 2013), when mobile genetic elements (MGE), carrying multiple antimicrobial determinants are involved, as explained elsewhere (Canton and Ruiz-Garbajosa, 2011).

As shown in Table 1, the 36 E. coli isolates carrying PMQR genes exhibited 18 different PFGE types. The $q n r \mathrm{~B}$ resistance genes (qnrB19 and $q n r B 5$ ) were present in different $E$. coli isolates classified in showed 15 PFGE types (A to O), demonstrating the dissemination of the resistance gene within a diverse bacterial population. Among the different PFGE types the major cluster "E" included 12 isolates producing $q n r B 19$. Two different PFGE types were found in the two $E$. coli isolates carrying qnrS1 (Q and R). The E. coli carrying $a a c\left(6^{\prime}\right)-I b$-cr belonged to 
Table 2

Phenotypic and genotypic characteristics of PMQR-harboring K. oxytoca, K. pneumoniae and E. fergusonii isolates from poultry.

\begin{tabular}{|c|c|c|c|c|c|c|}
\hline Isolates & Species identified & Farms & Resistance & PMQR gene & Plasmid family & PFGE \\
\hline $121 \mathrm{a}$ & K. oxytoca & 2 & AMC, CTX, CAZ, FOX, NAL, CIP, LEV, TET, CHL & $q n r \mathrm{~B} 19$ & ColE, FIB, F & Ko A \\
\hline $137 \mathrm{a}$ & K. oxytoca & 2 & AMC, CTX, CAZ, ATM, FOX, FEP, NAL, CIP, LEV, TET, SXT, CHL & qnrB19 & ColE, I1 & Ko B \\
\hline $108 \mathrm{a}$ & K. pneumoniae & 2 & AMC, CTX, NAL, CIP, LEV, TET, SXT, CHL & qnrB19 & $\overline{\mathrm{ColE}}$ & Kp A \\
\hline $117 \mathrm{c}$ & E. fergusonii & 2 & AMC, CTX, NAL, CIP, LEV, TET, SXT & qnrB19 & $\overline{\mathrm{ColE}}, \mathrm{I}, \mathrm{F}$ & Ef A \\
\hline $123 a$ & E. fergusonii & 2 & AMC, CTX, NAL, CIP, LEV, TET, CHL & qnrB19 & ColE, HI1, I1, F & Ef A \\
\hline $151 c$ & E. fergusonii & 2 & AMC, CTX, NAL, CIP, LEV, TET, SXT, CHL & qnrB19 & ColE, HI1, I1, F & Ef A \\
\hline $156 b$ & E. fergusonii & 2 & AMC, CTX, NAL, CIP, LEV, TET, SXT & qnrB19 & $\overline{\mathrm{ColE}}, \mathrm{HI} 1, \mathrm{I} 1, \mathrm{~F}$ & Ef A \\
\hline $157 b$ & E. fergusonii & 2 & AMC, CTX, CAZ, NAL, CIP, LEV, TET & qnrB19 & ColE, HI1, I1, F & Ef A \\
\hline $169 b$ & E. fergusonii & 2 & AMC, CTX, NAL, CIP, LEV, TET, SXT & qnrB19 & ColE, $\mathrm{I} 1, \mathrm{~F}$ & Ef A \\
\hline $171 b$ & E. fergusonii & 2 & AMC, CTX, NAL, CIP, LEV, TET, SXT & qnrB19 & ColE, I1, F & Ef A \\
\hline
\end{tabular}

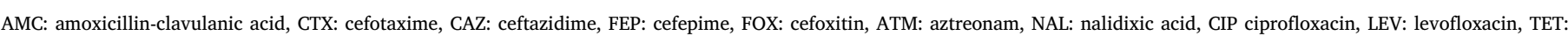
tetracycline, CHL: chloramphenicol, SXT: trimethoprim-sulfamethoxazole, GEN: gentamicin. The plasmids that carried PMQR genes are underlined.

a different PFGE type (P). Results in Table 2, show that all $E$. fergusonii carrying qnrB19 belonged to the same PFGE type (Ef A). Although, the two Klebsiella oxytoca isolates carrying qnrB19 were from different PFGE types (Table 2). The evaluation of the resistant $E$. coli population from farm 1 and farm 2 shows nine different PFGE types in each farm, carrying the resistance genes, without any common PFGE type shared between farms. These findings suggest that the dissemination of the resistance genes is most likely associated to MGE (e.g. plasmids), exchanged among different isolates, rather than with a unique resistant isolate prevailing. The phylogenetic groups $\mathrm{A}, \mathrm{B} 1$, and D were found among the $E$. coli harboring PMQR genes. Most isolates were identified in the phylogenetic group A (50\%), other $42 \%$ of the isolates were classified in the phylogenetic group D and $8 \%$ of the isolates were identified phylogenetic group B1 (Table 1). None of the isolates belonged phylogenetic group B2. In addition, 14 qnrB19-producing belonged to phylogenetic group D. Based on the phylogenetic analysis and PFGE, the population structure of $E$. coli isolates was diverse, even within the same poultry farm, characterizing a non-clonal dissemination and demonstrating the potential of these genes and MGEs to be maintained by different $E$. coli populations. Isolates within phylogenetic group B2 reported, as the most virulent, frequently causing infections were not detected. Nevertheless, among the phylogenetic groups identified in the present work, group D has also been involved in extraintestinal infections, causing concerns in clinical settings. The phylogenetic groups A and B1 are considered less virulent, however, were also described in human extraintestinal infections (Pitout, 2012).

As shown in Table 1, in all isolates, the qnrB and $q n r S$ genes were harboured on ColE-like plasmids. Moreover, $43 \mathrm{E}$. coli isolates carrying qnrB in ColE-like plasmids, also carried other replicon types, including IncHI1 $(n=1)$, IncI1 $(n=2)$, IncFIA $(n=3)$, IncFIB $(n=17)$, IncFIC $(\mathrm{n}=2)$, IncF $(\mathrm{n}=22)$, IncK $(\mathrm{n}=12)$ and $\operatorname{IncB} / \mathrm{O}(\mathrm{n}=2)$. E. coli isolates harboring qnrS1 showed the presence of IncHI1 $(\mathrm{n}=1)$, IncI1 $(\mathrm{n}=2)$, IncFIB $(\mathrm{n}=2) \operatorname{IncF}(\mathrm{n}=2)$ and IncA/C $(\mathrm{n}=1)$. The $E$. coli isolate carrying aac (6')-Ib-cr showed IncFIB, IncF, and IncY, however it was not possible to determine which plasmid harboured this gene. As shown in Table 2, E. fergusonii isolates carrying qnrB gene in ColE-like plasmids, also harboured IncHI1 $(n=4)$, IncI1 $(n=7)$ and IncF $(\mathrm{n}=8) . K$. oxytoca isolates harboring $q n r \mathrm{~B}$ also showed the replicon types IncFIB $(n=2)$, IncI1 $(n=7)$ and IncF $(n=8)$.

The Southern blot hybridization using the $S 1$-PFGE revealed that the $q n r B 19$ and $q n r B 5$ genes were located on low-molecular weight plasmids, smaller than $10 \mathrm{~Kb}$. The plasmids sequences from isolates carrying qnrB19 gene were analysed using GenBank database tools, and 100\% identity with plasmid pPAB19-3 was found (GenBank accession number JN985534). The sequenced plasmids were determined as ColE-like plasmids and the size was $2989 \mathrm{bp}$. The ColE-like backbone found included regions for plasmid replication and mobilization. The $q n r$ region was located near the Xer specific recombination site. The ISEcp1 was found in these plasmids downstream from $p s p$ gene (activator of the stress-inducible) and from $q n r$ gene. Moreover, the presence of this
MGE in different poultry Farms, bacterial species and E. coli isolates of different PFGE-types and phylogenetic groups, suggests the evidence of lateral gene transfer.

The ColE plasmids identified in all our isolates shared the same genetic sequence as those reported in E. coli from hospital, in Argentina, which has been circulating since 2007 in that country (Tran et al., 2012). However, now we report this replicon associated to PMQR determinants in isolates from food-producing poultry in Brazil, suggesting the capacity to disseminate. Moreover, it has been proposed that this RCR plasmid arrangement could play a role in the evolution of plasmids and present a model for DNA swapping between plasmid DNA mediated by site-specific recombination events at oriT and a Xer target site (Tran et al., 2012). The plasmids pPAB19-1, pPAB19-2, pPAB19-3 (pPAB19-3 also detected here), and pPAB19-4 share extensive homology among themselves and with other previously described small qnrB19-harboring plasmids (Tran et al., 2012).

Overall, as summarized in Tables 1 and 2, a high prevalence of Enterobacteriaceae, including E. coli, E. fergusonii and $K$. oxytoca, were found carrying PMQR determinant genes harboured in ColE-like plasmids. Moreover, these isolates also harboured other important replicon types. The increased resistance to quinolones and fluoroquinolones, determined by PMQR genes, was noticed in isolates that also showed resistance to other important antibiotics such as AMC, CTX, CAZ, ATM, GEN, CHL, TET. These characteristics were present in a diverse population of E. coli, as shown by PFGE and phylogeny, demonstrating that this dissemination of resistance has a non-clonal nature. The concern about the dissemination of PMQR genes is that these genes may facilitate the selection of higher levels of quinolone resistance (Jacoby et al., 2014). It is difficult to determine the incidence and prevalence of this plasmid carrying these genes, however, silent dissemination may be occurring in many ecological settings and in different countries in South America.

\section{Conclusion}

A high prevalence of PMQR determinants was found in Enterobacteriaceae isolated from healthy chickens. Different Enterobacteriaceae harboured these genes and the genes were found in two poultry Farms, showing the dissemination of resistant bacteria in food-producing animals, which may play an important role in the current increased prevalence of MDR bacteria in different environments reported worldwide. Therefore, the surveillance of resistant genes in the food chain is considered a key point to assist in the control of antimicrobial resistance.

\section{Conflict of interest statement}

None to declare. 


\section{Acknowledgements}

We would like to thank DVM, Mark Ishi, who contributed for sampling in poultry Farms, Dr. Luke Richards for his kind review of the text and São Paulo Research Foundation (FAPESP) for the constant support for our research (Grant no. 2014/14494-8). Rafael Penha Filho, (FAPESP, grant 2016/12293-0), São Paulo Research Foundation (FAPESP). L.N.A. was supported by post-doctoral fellowship, from National Council for Scientific and Technological Development (CNPq/ PNPD 2015).

\section{References}

Bardon, J., Husickova, V., Chroma, M., Kolar, M., 2013. Prevalence and characteristics of Escherichia coli strains producing extended-spectrum beta-lactamases in slaughtered animals in the Czech Republic. J. Food Prot. 76, 1773-1777.

Ben Sallem, R., Ben Slama, K., Rojo-Bezares, B., Porres-Osante, N., Jouini, A., Klibi, N., Boudabous, A., Saenz, Y., Torres, C., 2014. IncI1 plasmids carrying bla $a_{(\mathrm{CTX}-\mathrm{M}-1)}$ or bla $_{(\mathrm{CMY}-2)}$ genes in Escherichia coli from healthy humans and animals in Tunisia. Microb. Drug Resist. 20, 495-500.

Bolano, A., Stinchi, S., Preziosi, R., Bistoni, F., Allegrucci, M., Baldelli, F., Martini, A., Cardinali, G., 2001. Rapid methods to extract DNA and RNA from Cryptococcus neoformans. FEMS Yeast Res. 1, 221-224.

Canton, R., Ruiz-Garbajosa, P., 2011. Co-resistance: an opportunity for the bacteria and resistance genes. Curr. Opin. Pharmacol. 11, 477-485.

Carattoli, A., 2013. Plasmids and the spread of resistance. Int. J. Med. Microbiol. 303, 298-304.

Carattoli, A., Bertini, A., Villa, L., Falbo, V., Hopkins, K.L., Threlfall, E.J., 2005. Identification of plasmids by PCR-based replicon typing. J. Microbiol. Methods 63, 219-228.

Cattoir, V., Nordmann, P., 2009. Plasmid-mediated quinolone resistance in gram-negative bacterial species: an update. Curr. Med. Chem. 16, 1028-1046.

Cattoir, V., Poirel, L., Rotimi, V., Soussy, C.J., Nordmann, P., 2007. Multiplex PCR for detection of plasmid-mediated quinolone resistance qnr genes in ESBL-producing enterobacterial isolates. J. Antimicrob. Chemother. 60, 394-397.

Clemente, L., Manageiro, V., Jones-Dias, D., Correia, I., Themudo, P., Albuquerque, T., Geraldes, M., Matos, F., Almendra, C., Ferreira, E., Canica, M., 2015. Antimicrobial susceptibility and oxymino-beta-lactam resistance mechanisms in Salmonella enterica and Escherichia coli isolates from different animal sources. Res. Microbiol. 166, 574-583.

Clermont, O., Bonacorsi, S., Bingen, E., 2000. Rapid and simple determination of the Escherichia coli phylogenetic group. Appl. Environ. Microbiol. 66, 4555-4558.

CLSI, 2002. Performance Standards for Antimicrobial Disk and Dilution Susceptibility Tests for Bacteria Isolated from Animals; Approved Standard-Second Edition. CLSI Document M31-A2.

CLSI, 2013. Performance Standards for Antimicrobial Susceptibility Testing; TwentyThird Informational Supplement. CLSI Document M100-S23 Clinical and Laboratory Standards Institute, Wayne, PA.

Cunha, M.P., Lincopan, N., Cerdeira, L., Esposito, F., Dropa, M., Franco, L.S., Moreno, A.M., Knobl, T., 2017. Coexistence of CTX-M-2, CTX-M-55, CMY-2, FosA3, and QnrB19 in extraintestinal pathogenic Escherichia coli from poultry in Brazil. Antimicrob. Agents Chemother. 61.

Dalhoff, A., 2012. Resistance surveillance studies: a multifaceted problem-the fluoroquinolone example. Infection 40, 239-262.

Dionisi, A.M., Lucarelli, C., Owczarek, S., Luzzi, I., Villa, L., 2009. Characterization of the plasmid-borne quinolone resistance gene qnrB19 in Salmonella enterica serovar typhimurium. Antimicrob. Agents Chemother. 53, 4019-4021.

Fortini, D., Fashae, K., Garcia-Fernandez, A., Villa, L., Carattoli, A., 2011. Plasmidmediated quinolone resistance and beta-lactamases in Escherichia coli from healthy animals from Nigeria. J. Antimicrob. Chemother. 66, 1269-1272.

Gagliotti, C., Buttazzi, R., Sforza, S., Moro, M.L., 2008. Resistance to fluoroquinolones and treatment failure/short-term relapse of community-acquired urinary tract infections caused by Escherichia coli. J. Inf. Secur. 57, 179-184.

Garcia-Fernandez, A., Fortini, D., Veldman, K., Mevius, D., Carattoli, A., 2009. Characterization of plasmids harbouring qnrS1, qnrB2 and qnrB19 genes in Salmonella. J. Antimicrob. Chemother. 63, 274-281.

Harada, K., Asai, T., 2010. Role of antimicrobial selective pressure and secondary factors on antimicrobial resistance prevalence in Escherichia coli from food-producing animals in Japan. J Biomed Biotechnol 2010, 180682.

Ingram, P.R., Rogers, B.A., Sidjabat, H.E., Gibson, J.S., Inglis, T.J., 2013. Co-selection may explain high rates of ciprofloxacin non-susceptible Escherichia coli from retail poultry reared without prior fluoroquinolone exposure. J. Med. Microbiol. 62, 1743-1746.

Jacoby, G.A., Strahilevitz, J., Hooper, D.C., 2014. Plasmid-mediated quinolone resistance. Microbiol. Spectr. 2.
Jurado, S., Medina, A., de la Fuente, R., Ruiz-Santa-Quiteria, J.A., Orden, J.A., 2015. Resistance to non-quinolone antimicrobials in commensal Escherichia coli isolates from chickens treated orally with enrofloxacin. Jpn. J. Vet. Res. 63, 195-200.

Karczmarczyk, M., Martins, M., McCusker, M., Mattar, S., Amaral, L., Leonard, N., Aarestrup, F.M., Fanning, S., 2010. Characterization of antimicrobial resistance in Salmonella enterica food and animal isolates from Colombia: identification of a qnrB19-mediated quinolone resistance marker in two novel serovars. FEMS Microbiol. Lett. 313, 10-19.

Liebana, E., Carattoli, A., Coque, T.M., Hasman, H., Magiorakos, A.P., Mevius, D., Peixe, L., Poirel, L., Schuepbach-Regula, G., Torneke, K., Torren-Edo, J., Torres, C., Threlfall, J., 2013. Public health risks of enterobacterial isolates producing extendedspectrum beta-lactamases or AmpC beta-lactamases in food and food-producing animals: an EU perspective of epidemiology, analytical methods, risk factors, and control options. Clin. Infect. Dis. 56, 1030-1037.

Literak, I., Reitschmied, T., Bujnakova, D., Dolejska, M., Cizek, A., Bardon, J., Pokludova, L., Alexa, P., Halova, D., Jamborova, I., 2013. Broilers as a source of quinoloneresistant and extraintestinal pathogenic Escherichia coli in the Czech Republic. Microb. Drug Resist. 19, 57-63.

Lopez-Cadenas, C., Sierra-Vega, M., Garcia-Vieitez, J.J., Diez-Liebana, M.J., SahagunPrieto, A., Fernandez-Martinez, N., 2013. Enrofloxacin: pharmacokinetics and metabolism in domestic animal species. Curr. Drug Metab. 14, 1042-1058.

Magiorakos, A.P., Srinivasan, A., Carey, R.B., Carmeli, Y., Falagas, M.E., Giske, C.G., Harbarth, S., Hindler, J.F., Kahlmeter, G., Olsson-Liljequist, B., Paterson, D.L., Rice, L.B., Stelling, J., Struelens, M.J., Vatopoulos, A., Weber, J.T., Monnet, D.L., 2012. Multidrug-resistant, extensively drug-resistant and pandrug-resistant bacteria: an international expert proposal for interim standard definitions for acquired resistance. Clin. Microbiol. Infect. 18, 268-281.

Minarini, L.A., Poirel, L., Cattoir, V., Darini, A.L., Nordmann, P., 2008. Plasmid-mediated quinolone resistance determinants among enterobacterial isolates from outpatients in Brazil. J. Antimicrob. Chemother. 62, 474-478.

Oh, J.Y., Kwon, Y.K., Tamang, M.D., Jang, H.K., Jeong, O.M., Lee, H.S., Kang, M.S., 2016 Plasmid-mediated quinolone resistance in Escherichia coli isolates from wild birds and chickens in South Korea. Microb. Drug Resist. 22, 69-79.

Pallecchi, L., Riccobono, E., Sennati, S., Mantella, A., Bartalesi, F., Trigoso, C., Gotuzzo, E., Bartoloni, A., Rossolini, G.M., 2010. Characterization of small ColE-like plasmids mediating widespread dissemination of the qurB19 gene in commensal enterobacteria. Antimicrob. Agents Chemother. 54, 678-682.

Pallecchi, L., Riccobono, E., Mantella, A., Fernandez, C., Bartalesi, F., Rodriguez, H. Gotuzzo, E., Bartoloni, A., Rossolini, G.M., 2011. Small qnrB-harbouring ColE-like plasmids widespread in commensal enterobacteria from a remote Amazonas population not exposed to antibiotics. J. Antimicrob. Chemother. 66, 1176-1178.

Pitout, J.D., 2012. Extraintestinal pathogenic Escherichia coli: a combination of virulence with antibiotic resistance. Front. Microbiol. 3, 9.

Poirel, L., Cattoir, V., Nordmann, P., 2012. Plasmid-mediated quinolone resistance; interactions between human, animal, and environmental ecologies. Front. Microbiol. 3,24 .

Rodriguez-Martinez, J.M., Cano, M.E., Velasco, C., Martinez-Martinez, L., Pascual, A., 2011. Plasmid-mediated quinolone resistance: an update. J. Infect. Chemother. 17 , $149-182$.

Sambrook, J., Fritsch, E., Maniatis, T., 1989. Molecular Cloning: A Laboratory Manual, 2nd ed. Cold Spring Harbor Laboratory Press, New York.

Strahilevitz, J., Jacoby, G.A., Hooper, D.C., Robicsek, A., 2009. Plasmid-mediated quinolone resistance: a multifaceted threat. Clin. Microbiol. Rev. 22, 664-689.

Szmolka, A., Fortini, D., Villa, L., Carattoli, A., Anjum, M.F., Nagy, B., 2011. First report on $I n c \mathrm{~N}$ plasmid-mediated quinolone resistance gene qnrS1 in porcine Escherichia coli in Europe. Microb. Drug Resist. 17, 567-573.

Tamang, M.D., Nam, H.M., Kim, A., Lee, H.S., Kim, T.S., Kim, M.J., Jang, G.C., Jung, S.C., Lim, S.K., 2011. Prevalence and mechanisms of quinolone resistance among selected nontyphoid Salmonella isolated from food animals and humans in Korea. Foodborne Pathog. Dis. 8, 1199-1206.

Tran, T., Andres, P., Petroni, A., Soler-Bistue, A., Albornoz, E., Zorreguieta, A., ReyesLamothe, R., Sherratt, D.J., Corso, A., Tolmasky, M.E., 2012. Small plasmids harboring qnrB19: a model for plasmid evolution mediated by site-specific recombination at oriT and Xer sites. Antimicrob. Agents Chemother. 56, 1821-1827.

Vanni, M., Meucci, V., Tognetti, R., Cagnardi, P., Montesissa, C., Piccirillo, A., Rossi, A.M., Di Bello, D., Intorre, L., 2014. Fluoroquinolone resistance and molecular characterization of gyrA and parC quinolone resistance-determining regions in Escherichia coli isolated from poultry. Poult. Sci. 93, 856-863.

Wang, M., Guo, Q., Xu, X., Wang, X., Ye, X., Wu, S., Hooper, D.C., 2009. New plasmidmediated quinolone resistance gene, qnrC, found in a clinical isolate of Proteus mirabilis. Antimicrob. Agents Chemother. 53, 1892-1897.

Woerther, P.L., Burdet, C., Chachaty, E., Andremont, A., 2013. Trends in human fecal carriage of extended-spectrum beta-lactamases in the community: toward the globalization of CTX-M. Clin. Microbiol. Rev. 26, 744-758.

Zawack, K., Li, M., Booth, J.G., Love, W., Lanzas, C., Grohn, Y.T., 2016. Monitoring antimicrobial resistance in the food supply chain and its implications for FDA policy initiatives. Antimicrob. Agents Chemother. 60, 5302-5311. 